\title{
Dynamic Term-Modal Logic for Epistemic Social Network Dynamics
}

\section{Liberman, Andrés Occhipinti; Rendsvig, Rasmus K.}

\section{Published in:}

Proceedings of 7th International Workshop on Logic, Rationality, and Interaction

Link to article, DOI:

10.1007/978-3-662-60292-8_13

Publication date:

2019

Document Version

Early version, also known as pre-print

Link back to DTU Orbit

Citation $(A P A)$ :

Liberman, A. O., \& Rendsvig, R. K. (2019). Dynamic Term-Modal Logic for Epistemic Social Network Dynamics. In P. Blackburn, E. Lorini, \& M. Guo (Eds.), Proceedings of 7th International Workshop on Logic, Rationality, and Interaction (pp. 168-182). Springer. Lecture Notes in Computer Science (including subseries Lecture Notes in Artificial Intelligence and Lecture Notes in Bioinformatics) Vol. 11813 LNCS https://doi.org/10.1007/978-3-66260292-8 13

\section{General rights}

Copyright and moral rights for the publications made accessible in the public portal are retained by the authors and/or other copyright owners and it is a condition of accessing publications that users recognise and abide by the legal requirements associated with these rights.

- Users may download and print one copy of any publication from the public portal for the purpose of private study or research.

- You may not further distribute the material or use it for any profit-making activity or commercial gain

- You may freely distribute the URL identifying the publication in the public portal 


\title{
Dynamic Term-Modal Logic for Epistemic Social Network Dynamics (Extended Version)
}

\author{
Andrés Occhipinti Liberman ${ }^{1}$ and Rasmus K. Rendsvig ${ }^{2}$ \\ 1 DTU Compute \\ aocc@dtu.dk \\ 2 Center for Information and Bubble Studies, University of Copenhagen \\ rasmus@hum.ku.dk
}

\begin{abstract}
Logics for social networks have been studied in recent literature. This paper presents a framework based on dynamic term-modal logic (DTML), a quantified variant of dynamic epistemic logic (DEL). In contrast with DEL where it is commonly known to whom agent names refer, DTML can represent dynamics with uncertainty about agent identity. We exemplify dynamics where such uncertainty and de re/de dicto distinctions are key to social network epistemics. Technically, we show that DTML semantics can represent a popular class of hybrid logic epistemic social network models. We also show that DTML can encode previously discussed dynamics for which finding a complete logic was left open. As complete reduction axioms systems exist for DTML, this yields a complete system for the dynamics in question.
\end{abstract}

Keywords: social networks, term-modal logic, dynamic epistemic logic

\section{Introduction}

Over recent years, several papers have been dedicated to logical studies of social networks, their epistemics and dynamics [2,10-14,18-22,24,25]. The purpose of this literature typically is to define and investigate some social dynamics with respect to e.g. long-term stabilization or other properties, or to introduce formal logics that capture some social dynamics, or both.

This paper illustrates how dynamic term-modal logic (DTML, [1]) may be used for the second purpose. In general, term-modal logics are first-order modal logics where the index of modal operators are first-order terms. I.e., the operators double as predicates to the effect that e.g. $\exists x K_{x} N(x, a)$ is a formula-read, in this paper, as "there there exists an agent that knows of itself that it is a social network neighbor of $a$ ". The dynamic term-modal logic of [1] extends termmodal logic with suitably generalized action models that can effectuate both factual changes (e.g. to the network structure) as well as epistemic changes. For all the DTML action model encodable dynamics, [1] presents a general sound and complete reduction axiom-based logic in the style of dynamic epistemic logic (DEL, [3, 4]). Hence, whenever an epistemic social network dynamics is encodable using DTML, completeness follows. With this in mind, the main goal 
of this paper is to introduce and illustrate DTML as a formalism for representing epistemic social network dynamics, and to show how it may be used to obtain completeness results.

To this end, the paper progresses as follows. Sec. 2 sketches some common themes in the logical literature on social networks before introducing DTML and its application to epistemic social networks. Sec. 2 contains the bulk of the paper, with numerous examples of both static DTML models and action models. The examples are both meant to showcase the scope of DTML and to explain the more non-standard technical details involved in calculating updated models. In Sec. 3, we turn to technical results, where it is shown that DTML may encode popular static hybrid logical models of epistemic networks, as well as the dynamics of [12], for which finding a complete logic was left open. Sec. 4 contains final remarks.

\section{Models and Languages for Epistemic Social Networks}

To situate DTML in the logical literature on social networks, we cannot but describe the literature in broad terms. We omit both focus, formal details and main results of the individual contributions in favor of a broad perspective. That said, then all relevant literature in one way or other concern social networks. In general, a social network is a graph $(A, N)$ where $A$ is a set of agents and $N \subseteq A \times A$ is represents a social relation, e.g., being friends on some social media platform. Depending on interpretation, $N$ may be assumed irreflexive and symmetric. Social networks may be augmented with assignments of atomic properties to agents, representing e.g. behaviors, opinions or beliefs. One set of papers investigates such models and their dynamics using fully propositional static languages [13, 20,24,25].

A second set of papers combines social networks with a semantically represented epistemic dimension in the style of epistemic logic. In these works, the fundamental structure of interest is (akin to) a tuple

$$
\left(A, W,\left\{N_{w}\right\}_{w \in W}, \sim\right)
$$

with agents $A$ and worlds $W$, with each world $w$ associated with a network $N_{w} \subseteq$ $A \times A$, and $\sim: A \rightarrow \mathcal{P}(W \times W)$ associating each agent with an indistinguishability (equivalence) relation $\sim_{a}$. Call such a tuple an epistemic network structure.

The existing work on epistemic network structures may be organized in terms of the static languages they work with: propositional modal logic $[2,14]$ or hybrid logic $[9-12,18,19,21,22]$. In the former, the social network is described using designated atomic propositions (e.g., $N_{a b}$ for ' $b$ is a neighbor of $a^{\prime}$ ). To produce a model, an epistemic network structure is augmented with a propositional valuation $V: P \rightarrow \mathcal{P}(W)$. Semantically, $N_{a b}$ is then true at $w$ iff $(a, b) \in N_{w}$. Knowledge is expressed using operators $\left\{K_{a}\right\}_{a \in A}$ as in standard epistemic logic with $K_{a}$ the Kripke modality for $\sim_{a}$.

In the hybrid case, the network is instead described using modal operators. The hybrid languages typically include a set of agent nominals Nom (agent names), atoms $P$ and indexical modal operators $K$ and $N$, read "I know that" 
and "all my neighbors". Some papers additionally include state nominals, hybrid operators $\left(@_{x}, \downarrow_{x}\right)$ and/or universal modalities $U$ ("for all agents"). A hybrid network model is an epistemic network structures extended with two assignments: a nominal assignment $g: N o m \rightarrow A$ that names agents, and a two-dimensional hybrid valuation $V: P \rightarrow \mathcal{P}(W \times A)$, where $(w, a) \in V(p)$ represents that the indexical proposition $p$ holds of agent $a$ at $w$. The satisfaction relation is relative to both an epistemic alternative $w$ and an agent $a$, where the noteworthy clause are: $M, w, a \models p$ iff $(w, a) \in V(p) ; M, w, a \models K \varphi$ iff $M, v, a \models \varphi$ for every $v \sim_{a} w$; and $M, w, a \models N \varphi$ iff $M, w, b \models \varphi$ for every $b$ such that $N_{w}(a, b)$. With these semantics, formulas are read indexically. E.g. $K N p$ reads as "I know that all my neighbors are $p$ ".

In relation to these two language types, the term-modal approach of this paper lies closer to the former: By including a binary 'neighbor of' relation symbol $N$ in the signature of a term-modal language, the social network component of models is described non-modally. This straightforwardly allows expressing e.g. that that all agents know all their neighbors $\left(\forall x \forall y\left(N(x, y) \rightarrow K_{x}(N(x, y))\right)\right.$ or that an agent has de re vs. de dicto knowledge of someone being a neighbor $\left(\exists x K_{\underline{a}} N(\underline{a}, x)\right.$ vs. $\left.K_{\underline{a}} \exists x N(\underline{a}, x)\right)$. Moreover, hybrid languages can be translated into DTML, in such a way that hybrid formulas such as @ ${ }_{a} p$ ("agent $a$ has property $p$ ") become equivalent to $P(\underline{a})$, if $\underline{a}$ is the name of $a$.

\subsection{Term-Modal Logic and Epistemic Network Structures}

In general, term-modal languages may be based on any first-order signature, by for the purposes of representing social networks and factual properties of agents, we limit attention to the following: ${ }^{3}$

Definition 1. A signature is a tuple $\Sigma=(\mathrm{V}, \mathrm{C}, \mathrm{P}, N, \doteq)$ with $\mathrm{V}$ a countably infinite set of variables, $\mathrm{C}$ and $\mathrm{P}$ countable sets of constants and unary predicates, $N$ a binary relation symbol and $\doteq$ for identity. The terms of $\Sigma$ are $\mathrm{T}:=\mathrm{V} \cup \mathrm{C}$. With $t_{1}, t_{2} \in \mathrm{T}, x \in \mathrm{V}$ and $P \in \mathrm{P}$, the language $\mathcal{L}(\Sigma)$ is given by

$$
\varphi:=P\left(t_{1}\right)\left|N\left(t_{1}, t_{2}\right)\right|\left(t_{1} \doteq t_{2}\right)|\neg \varphi| \varphi \wedge \varphi\left|K_{t} \varphi\right| \forall x \varphi
$$

Standard Boolean connectives, $\top, \exists$ and $\hat{K}_{t}$ are defined per usual. With $\varphi \in$ $\mathcal{L}, t \in \mathrm{T}, x \in \mathrm{V}$, the result of replacing all occurrences of $x$ in $\varphi$ with $t$ is denoted $\varphi(x \mapsto t)$. Formulas from the first three clauses are called atoms; if an atom contains no variables, it is ground.

Throughout, $\underline{a}, \underline{b}$, etc. are used for constants and the relation symbol $N$ denotes a social network. The reading of $N\left(t_{1}, t_{2}\right)$ depends on application. $K_{t} \varphi$ is a term-indexed epistemic operator which read as "agent $t$ knows that $\varphi$ ". $\mathcal{L}(\Sigma)$ neither enforces nor requires a fixed-size agent set $A$, in contrast with standard epistemic languages, where the set of operators is given by reference to $A$. Hence the same language may be used to describe networks of varying size.

${ }_{3}$ The defined are special cases of the setting in [1], which allows general signatures and non-agent terms. [1] also reviews the term-modal literature. 
To interpret $\mathcal{L}(\Sigma)$, we use constant-domain models (the same number of agents in each world) with non-rigid constants (names, like predicates and relations, may change extension between worlds; this allows for uncertainty about agent identity). See Figs. 1 and 2 for examples of such models.

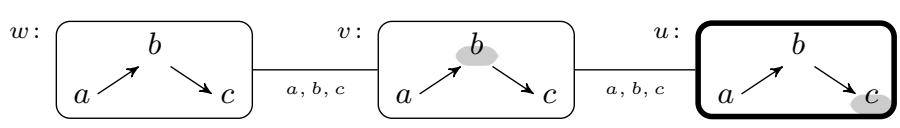

Fig. 1. Example 1, pt. 1 (Server Error). Three agents $a, b$ and $c$ work in a company with a hierarchical command structure, $\longrightarrow: a$ is the direct boss of $b$, who is the direct boss of $c$. The server has thrown an error after both $b$ and $c$ tampered with it. Either $w$ ) the server failed spontaneously, $v$ ) $b$ made a mistake (marked by gray) or $u$ ) $c$ made a mistake. Lines represent indistinguishability with reflexive and transitive links omitted. There is no uncertainty about the hierarchy, but nobody knows why the server failed. In fact, $c$ made a mistake: the actual world has a thick outline.

Definition 2. An $\mathcal{L}(\Sigma)$-model is a tuple $M=(A, W, \sim, I)$ where $A$ is a nonempty domain of agents, $W$ is a non-empty set of worlds, $\sim: A \rightarrow \mathcal{P}(W \times W)$ assigns to each agent $a \in A$ an equivalence relation on $W$ denoted $\sim_{a}$, and $I$ is an interpretation satisfying, for all $w \in W, 1$. for $c \in \mathrm{C}, I(c, w) \in A$; 2. for $P \in \mathrm{P}, I(P, w) \subseteq A ; 3 . I(N, w) \subseteq A \times A$. A pointed model is a pair $(M, w)$ with $w \in W$ called the actual world.

A variable valuation of $\Sigma$ over $M$ is a map $g: \mathrm{V} \rightarrow A$. The valuation identical to $g$ except mapping $x$ to $a$ is denoted $g[x \mapsto a]$. The extension of the term $t \in \mathrm{T}$ at $w$ in $M$ under $g$ is $\llbracket t \rrbracket_{w}^{I, g}=g(t)$ for $t \in \mathrm{V}$ and $\llbracket t \rrbracket_{w}^{I, g}=I(t, w)$ for $t \in \mathrm{C}$.

Given the inclusion of $N$ in the signature $\Sigma$, each $\mathcal{L}(\Sigma)$-model embeds an epistemic network structure $\left(A, W,\left(\sim_{a}\right)_{a \in A},(I(N, w))_{w \in W}\right)$.

Formulas are evaluated over pointed models using a direct combination of first-order and modal semantics:

Definition 3. Let $\Sigma, M$ and $g$ be given. The satisfaction of formulas of $\mathcal{L}(\Sigma)$ is given recursively by

$$
\begin{aligned}
& M, w \vDash_{g} P\left(t_{1}\right) \text { iff } \llbracket t_{1} \rrbracket_{w}^{I, g} \in I(P, w), \text { for } P \in \mathrm{P} . \\
& M, w \vDash_{g} N\left(t_{1}, t_{2}\right) \text { iff }\left(\llbracket t_{1} \rrbracket_{w}^{I, g}, \llbracket t_{2} \rrbracket_{w}^{I, g}\right) \in I(N, w) . \\
& M, w \vDash_{g}\left(t_{1} \doteq t_{2}\right) \text { iff } \llbracket t_{1} \rrbracket_{w}^{I, g}=\llbracket t_{2} \rrbracket_{w}^{I, g} . \\
& M, w \vDash_{g} \neg \varphi \text { iff not } M, w \vDash_{g} \varphi \text {. } \\
& M, w \vDash_{g} \varphi \wedge \psi \text { iff } M, w \vDash_{g} \varphi \text { and } M, w \vDash_{g} \psi . \\
& M, w \models_{g} \forall x \varphi \text { iff } M, w \models_{g[x \mapsto a]} \varphi \text { for all } a \in A . \\
& M, w \vDash_{g} K_{t} \varphi \text { iff } M, w^{\prime} \vDash_{g} \varphi \text { for all } w^{\prime} \text { such that } w \sim_{\llbracket t \rrbracket} \rrbracket_{w}^{I, g} w^{\prime} .
\end{aligned}
$$




\subsection{Knowing Who and Knowledge De Dicto and De Re}

First-order modal languages can represent propositional attitudes de dicto (about the statement) and de re (about the thing) in principled manners. For example, $K_{\underline{a}} \exists x P(x)$ is a de dicto statement: knowledge is expressed about the proposition that a $P$-thing exists. In contrast, $\exists x K_{\underline{a}} P(x)$ is a de re statement: it is expressed that of some thing $x$, that $x$ is known to be a $P$-thing. In general, de re statements are stronger than de dicto statements. The difference has been appreciated in epistemic logic since Hintikka's seminal [16], where he argues that $\exists x K_{\underline{a}}(x \doteq \underline{b})$ expresses that $a$ knows who $b$ is (see Fig. 2). Semantically, the formula ensures that the constant $\underline{b}$ refers to the same individual in all $\underline{a}$ 's epistemic alternatives (i.e., $\underline{b}$ is locally rigid). Both de dicto and de re statements may partially be expressed in propositional languages (e.g. de dicto $K_{a}\left(p_{b} \vee p_{c}\right)$ vs. de re $K_{a} p_{b} \vee K_{a} p_{c}$; see [2] for such a usage), but not in a principled manner: the required formulas will depend on the specific circumstances.

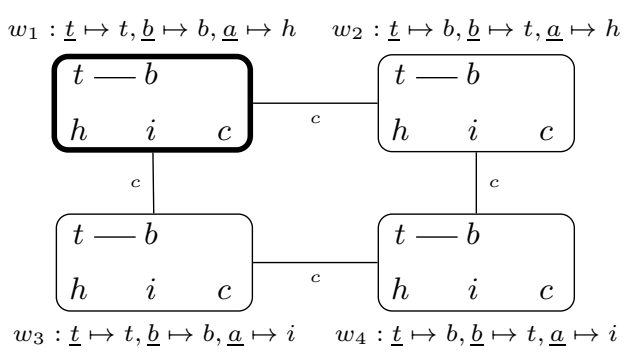

Fig. 2. Example 2, pt.1 (Knowing Who). Two thieves, $t$ and $b$, hide in a building with hostages $h$ and $i$. Outside, a cop, $c$, waits. To communicate safely, the thieves use code names 'Tokyo' and 'Berlin' for each other and 'The Asset' for the specially valuable hostage $h$. Agents $t, b, h$ and $i$ all know whom the code names denote (the names are rigid for them), but the cop does not. The code names are $\underline{t}$ for $t, \underline{b}$ for $b$ and $\underline{a}$ for $h$. Known by all, $h$ and $i$ are in fact called $\underline{h}$ and $\underline{i}$. The thief network (-) is assumed symmetric and transitive. The case is modeled using four worlds, identical up to code name denotation, (shown by $\mapsto$ ). E.g., in the actual world is $w_{1}, \underline{t}$ names $t$, but in $w_{4}$, it names $b$. Hence the cop does not know who Tokyo is: $M, w_{1} \vDash_{g} \neg \exists x K_{\underline{c}}(x \doteq \underline{t})$.

\subsection{Dynamics: Action Models and Product Update}

To code operations on static models, we use a a variant of DEL-style action models, adapted to term-modal logic (see Fig. 3). They include (adapted versions of) preconditions specifying when an event is executable ( $[3,4])$, postconditions describing the factual effects of events $([5,7,15])$ as well as edge-conditions representing how an agent's observation of an action depends on the agent's circumstances ( [6]) - for example their position in a network, cf. Fig. 3. Edgeconditions are non-standard and deserve a remark. With $E$ the set of events, edge-conditions are assigned by a map $Q$. For each edge $\left(e, e^{\prime}\right) \in E \times E, Q\left(e, e^{\prime}\right)$ is a formula with a single free variable $x^{\star}$. Given a model $M$, an agent $i$ cannot 
distinguish $e$ from $e^{\prime}$ iff the edge-condition $Q\left(e, e^{\prime}\right)$ is true in $M$ when the free variable $x^{\star}$ is mapped to $i$. Intuitively, if the situation described by the edgecondition is true for $i$, the way in which $i$ is observing the action does not allow her to tell whether $e$ or $e^{\prime}$ is taking place. See Figure 4 for an example. See [1] for a comparison of this approach to that of [6] and the term-modal action models of [17].

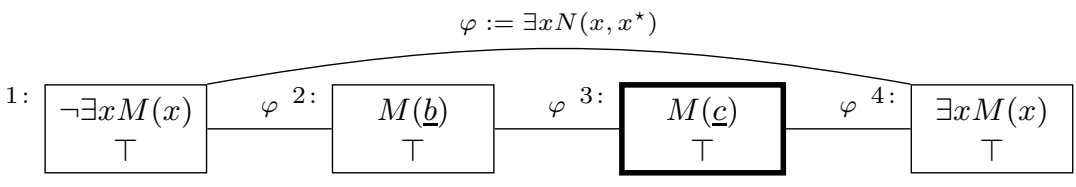

Fig. 3. Example 1, pt. 2 (Edge-Conditions: Announcement to Subgroup). To learn what happened to the server, the top boss $a$ requests its $\log$ file. The log holds one of four pieces of information: 1) Nobody made a mistake, 2) $b$ made a mistake $(M)$, 3) $c$ made a mistake or 4) somebody made a mistake. Each box represents one of these events: top lines are preconditions, bottom lines postconditions ( $T$ means no factual change). In fact, the $\log$ rats on $c$. $N$ denotes the hierarchy. The log is send only to the top boss: the others cannot see its content. This is represented by the edge-condition $\varphi$ : If you, $x^{\star}$, have a boss, then you cannot tell 1) from 2) nor 2) from 3) etc. For unillustrated edges, $Q(e, e)=\left(x^{\star} \doteq x^{\star}\right)$ and $Q\left(e, e^{\prime}\right)=\varphi$ when $e \neq e^{\prime}$.

For simplicity, we here only define action models that take pre-, post, and edge-conditions in the static language $\mathcal{L}(\Sigma)$. However, dynamic conditions are needed for completeness; we refer to [1] for details.

Definition 4. An action model for $\mathcal{L}(\Sigma)$ is a tuple $\Delta=(E, Q$, pre, post $)$ where

$\triangleright E$ is a non-empty, finite set of events.

$\triangleright Q:(E \times E) \rightarrow \mathcal{L}(\Sigma)$ where each edge-condition $Q\left(e, e^{\prime}\right)$ has exactly one free variable $x^{\star}$.

$\triangleright$ pre $: E \rightarrow \mathcal{L}(\Sigma)$ where each precondition pre $(e)$ has no free variables.

$\triangleright$ post $: E \rightarrow(\operatorname{GroundAtoms}(\mathcal{L}(\Sigma)) \rightarrow \mathcal{L}(\Sigma))$ assigns to each $e \in E$ a postcondition for each ground atom.

To preserve the meaning of equality, let $\operatorname{post}(e)(t \doteq t)=\top$ for all $e \in E$.

With no general restrictions on $Q$, to ensure that all agents' indistinguishability relations continue to be equivalence relations after updating, $Q$ must be chosen with care. Throughout, we assume $Q(e, e)=\left(x^{\star} \doteq x^{\star}\right)$ for all $e \in E$. To update, product update may be altered to fit the edge-condition term-modal setting as below. Fig. 4 illustrates the product update of Figs. 1 with 3. The use of postconditions is illustrated in Figs. 7 and 8. 
Definition 5. Let $M=(A, W, \sim, I)$ and $\Delta=(E, Q$, pre, post $)$ be given. The product update of $M$ and $\Delta$ is the model $M \otimes \Delta=\left(A^{\prime}, W^{\prime}, \sim^{\prime}, I^{\prime}\right)$ where

1. $A^{\prime}=A$

2. $W^{\prime}=\left\{(w, e) \in W \times E:(M, w) \vDash_{g}\right.$ pre $\left.(e)\right\}$ for any $g$,

3. $(w, e) \sim_{i}^{\prime}\left(w^{\prime}, e^{\prime}\right)$ iff $w \sim_{i} w^{\prime}$ and $M, w \vDash_{g\left[x^{\star} \mapsto i\right]} Q\left(e, e^{\prime}\right)$,

4. $I^{\prime}(c,(w, e))=I(c, w)$ for all $c \in \mathrm{C}$, and

$I^{\prime}(X,(w, e))=\left(I(X, w) \cup X^{+}(w)\right) \backslash X^{-}(w)$, for $X=\{P, N\}, P \in \mathrm{P}$, where:

$P^{+}(w):=\left\{\llbracket t \rrbracket_{w}^{I, v}:(M, w) \vDash_{g} \operatorname{post}(e)(P(t))\right\} ;$

$P^{-}(w):=\left\{\llbracket t \rrbracket_{w}^{I, v}:(M, w) \not_{g} \operatorname{post}(e)(P(t))\right\} ;$

$N^{+}(w):=\left\{\left(\llbracket t_{1} \rrbracket_{w}^{I, v}, \llbracket t_{2} \rrbracket_{w}^{I, v}\right):(M, w) \vDash_{g} \operatorname{post}(e)\left(N\left(t_{1}, t_{2}\right)\right)\right\} ;$

$N^{-}(w):=\left\{\left(\llbracket t_{1} \rrbracket_{w}^{I, v}, \llbracket t_{2} \rrbracket_{w}^{I, v}\right):(M, w) \not \not_{g} \operatorname{post}(e)\left(N\left(t_{1}, t_{2}\right)\right)\right\}$

If $(M, w) \models \operatorname{pre}(e)$, then $(A, e)$ is applicable to $(M, w)$, and the product update of the two is the pointed model $(M \otimes \Delta,(w, e))$. Else it is undefined.

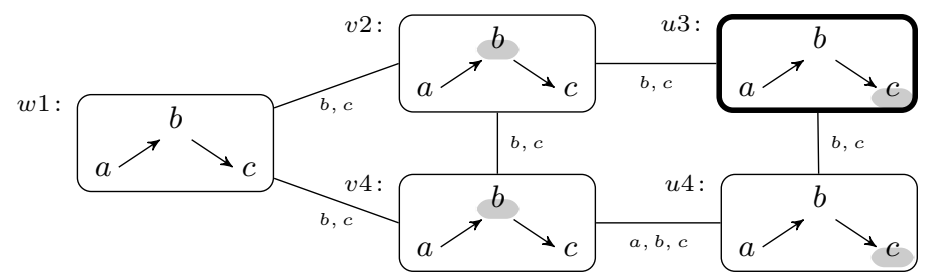

Fig. 4. Example 1, pt. 3 (Product Update: Edge-Conditions). The product update of Fig. 1 and Fig. 3. After checking the logs, the boss has learned that $c$ made a mistake, while both $b$ and $c$ are now both uncertain about this, as well as about the boss' information. Worlds are named using by the world-event pair they represent: $w 1$ is the child of $w$ and 1, etc. The pair $w 2$ is not a world: $w$ did not satisfy the precondition of 1 . We have $w 1 \sim_{b}^{\prime} v 2$ as $w \sim_{b} v$ and $M, w \vDash_{g\left[x^{\star} \mapsto a\right]} Q(1,2)$-as $M, w \vDash_{g} \exists x N(x, \underline{b})$. Likewise, $v 2 \sim_{b}^{\prime} w 1$ as $v \sim_{b} w$ and $M, v \vDash_{g} \exists x N(x, \underline{b})$. That $w 1 \chi_{a}^{\prime} v 2$ follows as $M, w \vDash_{g} \neg \exists x N(x, \underline{b})$, but $v 4 \sim_{a}^{\prime} u 4$ as $M, v \vDash_{g}(\underline{a} \doteq \underline{a})$. The same reason, reflexive loops are preserved. The boss now knows that $c$ made a mistake: $K_{\underline{a}} M(\underline{c})$.

\subsection{Announcements De Dicto and De Re}

With de dicto and de re statements expressible in DTML, they may be used to define principled announcements, as exemplified in Fig. 5 and 6. The action models are applicable to any DTML model for a signature that includes the constant $\underline{a}$ and the predicate $M$, irrespective of the size of the set of agents. This level of general applicability is not mirrored in standard DEL action models. 

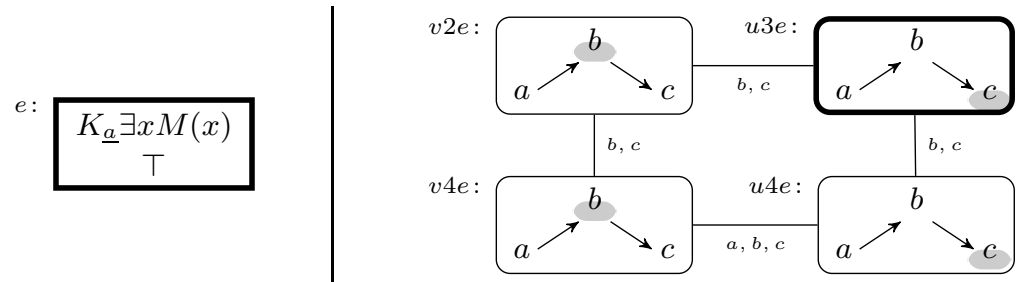

Fig. 5. Example 1, pt. 3 (De Dicto Announcement). The boss breaks the news from the $\log$ to $b$ and $c$ piecemeal. Left: First, $a$ makes a de dicto announcement: $a$ knows that somebody made a mistake. Right: The effect on Fig. 4. Only $w 1$ does not survive. In $u 3 e$, everybody knows de dicto that somebody messed up: $\forall x K_{x} \exists y M(y)$. The boss also knows de re, i.e., knows who: $u 3 e \vDash_{g} \exists x K_{a} M(x)$, as $u 3 e \vDash_{g[x \mapsto c]}$ $K_{a} M(x)$. The employees do not know that a knows de re: $u 3 e \vDash_{g} \forall x(\exists y N(y, x) \rightarrow$ $\left.\hat{K}_{x} \neg \exists z K_{a} M(z)\right)$ - since $v 4 e \vDash_{g} M(x)$ iff $g(x)=b$, but then $u 4 e \not \nvdash_{g} M(x)$. I.e., there is no one object to serve as valuation for $x$ such that $v 4 e$ and $u 4 e$ satisfy $M(x)$ simultaneously). The employees are held in suspense!
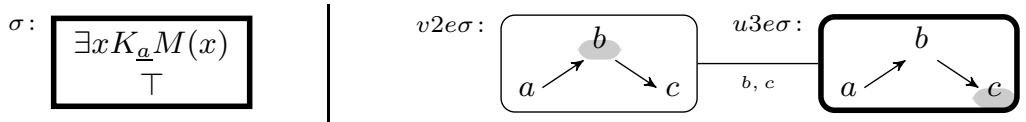

Fig. 6. Example 1, pt. 4 (De Re Announcement). Following a dramatic pause, the boss reveals a stronger piece of information: the boss knows who messed up. This de re announcement is on the left, with $Q(e, e)=\left(x^{\star}=x^{\star}\right)$; its result on Fig. 5 (Right) on the right. In $u 3 e \sigma$, everybody knows that $a$ has de re knowledge: $\forall x K_{x} \exists y K_{a} M(y)$, but $b$ and $c$ still only have de dicto knowledge: $\forall x\left((x=b \vee x=c) \rightarrow K_{x} \exists y M(y) \wedge\right.$ $\left.\neg \exists z K_{x} M(z)\right)$.

\subsection{Postconditions and Network Change}

Action models with postconditions allows DTML to represent changes to the social network. Such changes may be combined with the general functionality of action models such that some agents may know what changes occur while others remain in the dark. Fig. 7 provides a simple example, including the details calculating the updated network. Fig. 8 presents an example of how de re/de dicto knowledge affects what is learned by a publicly observed network change.
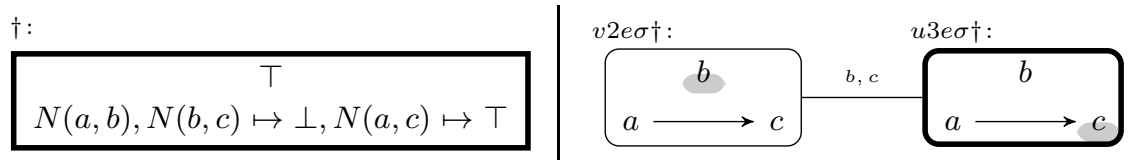

Fig. 7. Example 1, pt. 5 (Getting Fired). The employees are dying to know who messed up the server. But the boss just proclaims: ' $b$, you are fired! $c$, you are promoted!' Left: Action with three instructions for factual change: $\operatorname{post}(\dagger)(N(a, b))=\perp$, $\operatorname{post}(\dagger)(N(b, c))=\perp$ and post $(\dagger)(N(a, c))=\top$ (illustrated by $\mapsto)$. Else post $=i d$. As $u 3 e \sigma \not \models \perp$, the first two instructions entail that $(a, b),(b, c) \in N^{-}(u 3 e \sigma)$, while the latter implies that $(a, c) \in N^{+}(u 3 e \sigma)$. Right: The network is updated to $I^{\prime}(N, u 3 e \sigma \dagger)=$ $\left(I(N, u 3 e \sigma) \cup N^{+}(u 3 e \sigma)\right) \backslash N^{-}(u 3 e \sigma)=(\{(a, b),(b, c)\} \cup\{(a, c)\}) \backslash\{(a, b),(b, c)\}=$ $\{(a, b)\}$. In $u 3 e \sigma \dagger$, neither $b$ nor $c$ know who made the mistake. Unrepresented, $a$ thinks that only bad superiors let their employees make mistakes. 

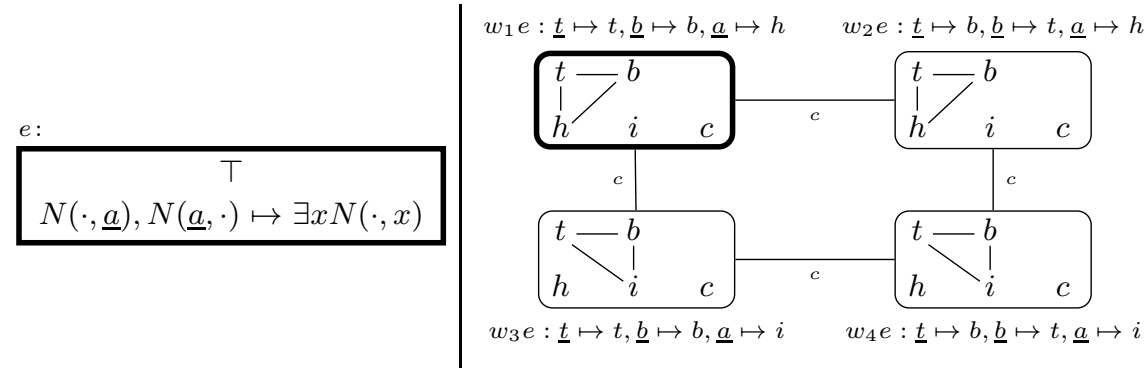

Fig. 8. Example 2, pt.2 (Becoming Criminal) Left: The thieves convince The Asset to cooperate with them, in exchange for stolen goods. For simplicity, assume that the action of $\underline{a}$ joining the thief network is noticed by everyone. We model this with the action model, with post $(e)(N(\cdot, \underline{a}))=\exists x N(\cdot, x)$ and post $(e)(N(\underline{a}, \cdot))=\exists x N(x, \cdot)$ for $\cdot \in\{\underline{t}, \underline{b}, \underline{a}, \underline{h}, \underline{i}, \underline{c}\}$. Informally, these say: "If you are a member of the network, then $\underline{a}$ becomes your neighbor". Right: The effect of event $e$ on Fig. 2: The network has changed in all worlds, but differently. E.g., in $w_{1}$, we had $\neg N(\underline{b}, \underline{a})$; in $\left(w_{1}, e\right)$, we have $N(\underline{b}, \underline{a})$ as $(b, h) \in N^{+}\left(\left(w_{1}, e\right)\right)$ since $w_{1} \vDash_{g} \operatorname{post}(e)(N(\underline{b}, \underline{a}))$-i.e., $\exists x N(\underline{b}, x)$. Now all thieves and hostages know the new network, as they know whom $\underline{a}$ refers to. E.g.: Tokyo knows all her neighbors, $\left(w_{1}, e\right) \vDash_{g} \forall x\left(N(\underline{t}, x) \rightarrow K_{\underline{t}} N(\underline{t}, x)\right)$. The cop only learns that some hostage has joined the network, but can't tell whom: $\left(w_{1}, e\right) \vDash_{g} K_{\underline{c}} \exists x(x \neq \underline{t} \wedge x \neq$ $\underline{b} \wedge N(\underline{t}, x))$ but $\left(w_{1}, e\right) \not \forall_{g} \exists x K_{\underline{c}}(x \neq \underline{t} \wedge x \neq \underline{b} \wedge N(\underline{t}, x))$.

\subsection{Learning Who}

Allowing for the possibility of non-rigid names has the consequence that public announcements of atomic propositions may differ in informational content depending on the epistemic state of the listener. This can be exploited by the thieves of Example 2 to enforce a form of privacy - as code names should. The notion of privacy involved is orthogonal to the notion of privacy modeled in DEL using private announcements. Though the message is public in the standard sense of everyone being aware of it and its content, as it involves non-rigid names, its epistemic effects are not the same for all agents. This is in contrast with standard public announcements, which yield the same information to everyone.
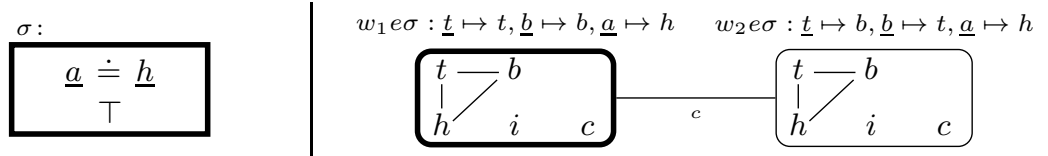

Fig. 9. Example 2, pt.4 (Revealing the Asset) In the model in Fig. 8 (Right), even a public announcement of $N(\underline{t}, \underline{a})$ would not inform the cop about who joined the network. To know who joined the network, the cop must learn who The Asset is. As the cop knows who $\underline{h}$ is, learning that $\underline{h}$ is The Asset suffices. Left: The event model $\sigma$ for the public announcement that $\underline{a} \doteq \underline{h}$, revealing the identity of The Asset. Right: The product update of Fig. 8 (Right) and event $\sigma$. The cop now knows the structure of the network, as a result of the removal of $w_{3} e$ and $w_{4} e$. 


\section{Embedding Dynamic Social Network Logics in DTML}

This section examines relations between the hybrid network models and their languages to DTML. As hybrid languages corresponds to fragments of first-order logic with equality $\left(\mathrm{FOL}_{=}\right)$, which term-modal logic extends, it stands to reason that the hybrid languages and models mentioned in Sec. 2 may be embedded in term-modal logic. A precise statement and a proof sketch follows below. Turning to dynamics, things are more complicated. [22] presents a very flexible hybrid framework expressing network dynamics using General Dynamic Dynamic Logic (GDDL, [23]). We leave general characterizations of equi-expressive fragments of GDDL and DTML as open question, but remark that all GDDL action-examples of [22] may be emulated using DTML action models, and in many cases via fairly simple ones. More thoroughly, we show that the logic of Knowledge, Diffusion and Learning (KDL, [12]) has a complete and decidable system, a question left open in [12]. This is shown by encoding KDL in DTML.

\subsection{Embedding Static Languages and Models}

The static hybrid languages of $[9-12,19,21,22]$ are all sub-languages of $\mathcal{L}(P, N o m)$, defined and translated into DTML below. [18] also includes state nominals, which our results do not cover. $\mathcal{L}(P, N o m)$ is read indexically, as described in Sec. 2.

Definition 6. With $p \in P$ and $x \in N$ om, the language $\mathcal{L}(P, N o m)$ is given by

$$
\varphi:=p|\neg \varphi| \varphi \wedge \varphi\left|@_{x} \varphi\right| K \varphi|N \varphi| U \varphi
$$

Denote the fragments without $U$ and $@_{x}$ by $\mathcal{L}_{-U}(P, N o m)$ and $\mathcal{L}_{-@}(P, N o m)$.

Hybrid logics may be translated into $\mathrm{FOL}_{=}$; our translation resembles that of [8]. We identify agent nominals with first-order variables, translate the modal operator $N$ to the relation symbol $N(\cdot, \cdot)$, and relativize the interpretation of the indexical $K$ to the nominal/variable $x$ by using the term-indexed operator $K_{x}$. Formally, the translation is defined as follows.

Definition 7. Let $\Sigma_{n}(P, N o m)=(\mathrm{V}, \mathrm{C}, \mathrm{P}, N, \doteq)$ be the signature with $\mathrm{V}=N o m$, $\mathrm{C}=\left\{\underline{a}_{1}, \ldots, \underline{a}_{n}\right\}$ and $\mathrm{P}=P$. Translations $T_{x}, T_{y}$ both mapping $\mathcal{L}(P, N o m)$ to $\mathcal{L}\left(\Sigma_{n}(P, N o m)\right)$ are defined by mutual recursion. It is assumed that two nominals $x$ and $y$ are given which do not occur in the formulas to be translated. For $p \in P$ and $i \in N o m$, define $T_{x}$ by:

$$
\begin{aligned}
T_{x}(p) & =p(x) & T_{x}\left(@_{i} \varphi\right) & =T_{x}(\varphi)(x \mapsto i) \\
T_{x}(i) & =x \doteq i & T_{x}(N \varphi) & =\forall y\left(N(x, y) \rightarrow T_{y}(\varphi)\right) \\
T_{x}(\varphi \wedge \psi) & =T_{x}(\varphi) \wedge T_{x}(\psi) & T_{x}(K \varphi) & =K_{x} T_{x}(\varphi) \\
T_{x}(\neg \varphi) & =\neg T_{x}(\varphi) & T_{x}(U \varphi) & =\forall x T_{x}(\varphi)
\end{aligned}
$$

The translation $T_{y}$ is obtained by exchanging $x$ and $y$ in $T_{x}$.

To show the translation truth-preserving, we embed the class of hybrid network models into a class of term-modal models: 
Definition 8. Let $M=\left(A, W,\left(N_{w}\right)_{w \in W}, \sim, g, V\right)$ be a hybrid network model for $\mathcal{L}(P, N o m)$. Then the TML image of $M$ is the $\mathcal{L}\left(\Sigma_{n}(P, N o m)\right) \mathrm{TML}$ model $\mathrm{T}(M)=(A, W, \sim, I)$ sharing $A, W$ and $\sim$ with $M$ and with $I$ given by

1. $\forall \underline{c} \in \mathrm{C}, \forall w, v \in W, \forall a, b \in A,\left(I(\underline{c}, w)=a\right.$ and $\left.w \sim_{b} v \Rightarrow I(\underline{c}, v)=a\right)$

2. $I(p, w)=\{a:(w, a) \in V(p)\}$

3. $I(N, w)=\left\{(a, b) \in A \times A:(a, b) \in N_{w}\right\}$

The model $\mathrm{T}(M)$ has the same agents, worlds and epistemic relations as $M$. The interpretation 1. encodes weak rigidity: if $(w, v) \in \bigcup_{a \in A} \sim_{a}$, then any constant denotes the same in $w$ and $v$, emulating the rigid names of hybrid network models; 2 . ensures predicates are true of the same agents at the same worlds, and 3. ensures the same agents are networked in the same worlds.

With the translations $T_{x}, T_{y}$ and the embedding $\mathrm{T}$, it may be shown that DTML can fully code the static semantics of $\mathcal{L}(P, N o m)$ hybrid network logics:

Proposition 1. Let $M=\left(A, W,\left(N_{w}\right)_{w \in W}, \sim, g, V\right)$ be a hybrid network model. Then for all $\varphi \in \mathcal{L}(P, N o m), M, w, g(\bullet) \models \varphi$ iff $\mathrm{\top}(M), w \models_{g} T_{\bullet}(\varphi), \bullet=x, y$.

\subsection{KDL Dynamic Transformations and Learning Updates in DTML}

We show that KDL [12] dynamics may be embedded in DTML, for finite agent sets (as assumed in [12]). Given Prop. 1, we argue that each KDL model transformer is representable by a DTML action model and that the dynamic KDL language is truth-preservingly translatable into a DTML sublanguage. The logic of the class of KDL models is, up to language translations, the logic of its corresponding class of DTML models. We show that the logic of this class of DTML models can be completely axiomatized, and the resulting system is decidable. Thus, by embedding KDL in DTML, we find a complete system for the former.

In $\mathrm{KDL}^{4}$, agents are described by feature propositions reading "for feature $\mathrm{f}$, I have value $z$ ". With $F$ a countable set of features and $Z_{f}$ a finite set of possible values of $f \in F$, the set of feature propositions is $F P=\left\{(f \doteqdot z): f \in F, z \in Z_{f}\right\}$. The static language of [12] is then $\mathcal{L}_{-U}(\mathrm{FP}$, Nom $)$. The dynamic language $\mathcal{L}_{\mathrm{KDL}}$ extends $\mathcal{L}_{-U}(\mathrm{FP}$, Nom $)$ with dynamic modalities $[d]$ and $[\ell]$ for dynamic transformations $d$ and learning updates $\ell$ :

$$
\varphi::=(\mathrm{f} \doteqdot \mathrm{z})|i| \neg \varphi|\varphi \wedge \varphi| @_{i} \varphi|N \varphi| K \varphi|[d] \varphi|[\ell] \varphi
$$

A dynamic transformation $d$ changes feature values of agents: each is a pair $d=(\Phi$, post $)$ where $\Phi \subseteq \mathcal{L}_{\mathrm{KDL}}$ is a non-empty finite set of pairwise inconsistent formulas and post : $\Phi \times \mathrm{F} \rightarrow\left(Z_{n} \cup\{\star\}\right)$ is a $\mathrm{KDL}$ post-condition. Encoded by $\operatorname{post}(\varphi, \mathrm{f})=x$ is the instruction: if $(w, a) \vDash \varphi$, then after $d$, set $\mathrm{f}$ to value $x$ at $(w, a)$, if $x \in Z_{n}$; if $x=\star, \mathrm{f}$ is unchanged. A learning update cuts accessibility relations: the update with finite $\ell \subseteq \mathcal{L}_{\mathrm{KDL}}$ keeps a $\sim_{a}$ link between worlds $w$ and $v$ iff, for all $\varphi \in \ell,(w, b) \vDash \varphi \Leftrightarrow(v, b) \vDash \varphi$ for all neighbors $b$ of $a$.

\footnotetext{
${ }^{4}$ Notation here is equivalent but different to fit better with the rest of this paper.
} 
Definition 9. Given a $\mathrm{KDL}$ model $M=\left(A, W,\left(N_{w}\right)_{w \in W}, \sim, g, V\right)$, the model reached after applying $d$ is $M^{d}=\left(A^{d}, W^{d},\left(N_{w}^{d}\right)_{w \in W}, \sim^{d}, g^{d}, V^{d}\right)$ where only $V^{d}$ is different, and is defined as follows: $(w, a) \in V^{d}(\mathrm{f} \doteqdot \mathrm{z})$ iff $(a) \operatorname{post}(\varphi, \mathrm{f})=x$ for some $\varphi \in \Phi$ such that $M, w, a \models \varphi$, where $x \neq \star$; or (b) condition (a) does not hold and $(w, a) \in V(\mathrm{f} \doteqdot \mathrm{z})$.

Definition 10. A learning update is a finite set of formulas $\ell \subseteq \mathcal{L}_{\mathrm{KDL}}$. Given $a \mathrm{KDL}$ model $M=\left(A, W,\left(N_{w}\right)_{w \in W},\left(\sim_{a}\right)_{a \in A}, g, V\right)$, the model after $\ell$ is $M^{\ell}=$ $\left(A, W,\left(N_{w}\right)_{w \in W},\left(\sim_{a}^{\prime}\right)_{a \in A}, g, V\right)$ where:

$w \sim_{a}^{\prime} v$ iff $w \sim_{a} v$ and $\forall b \in A\left(N_{w}(a, b) \Rightarrow \forall \varphi \in \ell(M, w, b \models \varphi\right.$ iff $\left.M, v, b \models \varphi)\right)$

Let $\mathrm{D}$ and $\mathrm{L}$ be the sets of dynamic transformations and learning updates. The result of applying $\dagger \in \mathrm{D} \cup \mathrm{L}$ to $M$ is denoted $M^{\dagger}$, and the [†] modality has semantics $M, w, a \models[\dagger] \varphi$ iff $M^{\dagger} w, a \models \varphi$.

As we show below, for every $\dagger \in \mathrm{D} \cup \mathrm{L}$, there is a pointed DTML action model $\Delta^{\dagger}$ with identical effects. As KDL operations may involve formulas with $[\dagger]$-modalities, we must use DTML action models that allow $[\Delta, e]$-modalities in their conditions, and translate $\mathcal{L}_{\mathrm{KDL}}$ into the general DTML language that results, denoted $\mathcal{L}\left(\Sigma_{n}(\mathrm{FP}, N o m)+[\Delta]\right) .{ }^{5}$ This language is interpreted over DTML models with standard action model semantics:

$$
(M, w) \vDash_{g}[\Delta, e] \varphi \text { iff } M \otimes \Delta,(w, e) \vDash \varphi .
$$

We define now the action models $\Delta^{\dagger}$. For a dynamic transformation $d \in \mathrm{D}$, [11] provide reduction axioms showing $d$ 's instructions statically encodable in $\left.\mathcal{L}_{\mathrm{KDL}}\right)$. The reduction axiom for atoms is as follows:

$$
[d] \mathrm{f} \doteqdot \mathrm{z} \leftrightarrow\left(\bigvee_{\varphi \in \Phi: \operatorname{post}(\varphi, \mathrm{f})=\mathrm{z}, \mathrm{z} \in \mathrm{Z}_{\mathrm{f}}} \varphi\right) \vee\left(\neg\left(\bigvee_{\varphi \in \Phi: \operatorname{post}(\varphi, \mathrm{f})=\mathrm{z}, \mathrm{z} \in \mathrm{Z}_{\mathrm{f}}} \varphi\right) \wedge \mathrm{f} \doteqdot \mathrm{z}\right)
$$

As $d$ changes atomic truth values under a definable instruction, its effects may be simulated by an action model with a matching post-condition (i.e., the translation of the definable instruction). More specifically, the action model $\Delta^{d}$ is defined as follows.

Definition 11. For dynamic transformation $d=(\Phi$, post $)$, the action model $\Delta^{d}=(E, Q$, pre, post $)$ is defined by $E=\left\{e^{d}\right\}, Q\left(e^{d}, e^{d}\right)=\operatorname{pre}\left(e^{d}\right)=\top$ and for each constant $\underline{a}, \operatorname{post}(e)\left(T_{x}(\mathrm{f} \doteqdot \mathrm{z})(x \mapsto \underline{a})\right)=$

$$
T_{x}\left(\left(\bigvee_{\varphi \in \Phi: p \operatorname{post}(\varphi, \mathrm{f})=\mathrm{z}, \mathrm{z} \in \mathrm{Z}_{\mathrm{f}}} \varphi\right) \vee\left(\neg\left(\bigvee_{\varphi \in \Phi: \operatorname{post}(\varphi, \mathrm{f})=\mathrm{z}, \mathrm{z} \in \mathrm{Z}_{\mathrm{f}}} \varphi\right) \wedge \mathrm{f} \doteqdot \mathrm{z}\right)\right)(x \mapsto \underline{a})
$$

For a learning update $\ell \in \mathrm{L}, \Delta^{\ell}$ has events $e^{X}, e^{Y}$ for any consistent subsets $X, Y$ of $\{\varphi(\underline{c}), \neg \varphi(\underline{c}): \varphi \in \ell, \underline{c} \in \mathrm{C}\}$ with edge-condition $Q\left(e^{X}, e^{Y}\right)$ satisfied for agents for whom all neighbors agree on $X$ and $Y$. Unsatisfied edge-conditions thereby capture the link cutting mechanism of $\ell$. The detailed definition of $\Delta^{\ell}$ is as follows.

\footnotetext{
${ }^{5}$ Defined using double recursion as standard; see [1] for details.
} 
Definition 12. Let $\ell=\left\{\varphi_{1}, \ldots, \varphi_{m}\right\}$ be a learning update. Let $T_{x}(\ell):=\left\{T_{x}\left(\varphi_{i}\right) \mid\right.$ $i=1, \ldots, n\}$ and let $G_{\ell}:=\left\{T_{x}(\varphi)(x \mapsto \underline{a}) \mid T_{x}(\varphi) \in T_{x}(\ell), \underline{a} \in \mathrm{C}\right\}$ be the grounding of $T_{x}(\ell)$ obtained by replacing each free occurrence of $x$ in $T_{x}(\varphi)$ for each possible constant $\underline{a} \in \mathrm{C}$. Define a $G_{\ell}$-valuation as a function val $: G_{\ell} \rightarrow\{0,1\}$ and let $\mathcal{V}_{\ell}$ be the set of all such valuations.

Definition 13. Let $\ell$ be a learning update. The corresponding DTML action model $\Delta^{\ell}=\left(E^{\ell}, Q^{\ell}\right.$, pre $^{\ell}$, post $\left.{ }^{\ell}\right)$ is defined by letting

$$
\begin{aligned}
& \triangleright E^{\ell}=\left\{e^{v a l} \mid v a l \in \mathcal{V}_{\ell}\right\} \text {, } \\
& \triangleright \operatorname{pre}^{\ell}\left(e^{v a l}\right)=\bigwedge\{\varphi \mid \operatorname{val}(\varphi)=1\} \cup\{\neg \varphi \mid \operatorname{val}(\varphi)=0\} \\
& \triangleright Q^{\ell}\left(e^{v a l}, e^{v a l}\right)=\top \\
& \triangleright Q^{\ell}\left(e^{v a l}, e^{v a l^{\prime}}\right)=\bigwedge_{\left\{\underline{a} \in \mathrm{C} \mid \exists \varphi \in \ell \text { s.t. } \operatorname{val}\left(T_{x}(\varphi)(x \mapsto \underline{a})\right) \neq v_{a l}\left(T_{x}(\varphi)(x \mapsto \underline{a})\right)\right\}} \neg N\left(x^{\star}, \underline{a}\right) \text {, for } \\
& \text { any two distinct events } e^{\text {val }}, e^{\text {val }} \\
& \triangleright \operatorname{post}^{\ell}(e)=i d \text { for all } e \in E^{\ell}
\end{aligned}
$$

Note that the signature $\Sigma_{n}(F P, A N o m)$ is defined to have finitely many constants $\mathrm{C}=\left\{\underline{a}_{1}, \ldots, \underline{a}_{n}\right\}$, and hence both $E$, the preconditions and the edgeconditions in $\Delta^{\ell}$ are finite, as required. The action model $\Delta^{\ell}$ works as follows. Each event $e^{v a l}$ corresponds to one way the agents can be with respect to $G_{\ell}$, as indicated by val. The edge conditions control how links get cut. Two worlds $\left(w, e^{v a l}\right)$ and $\left(v, e^{v a l^{\prime}}\right)$ in the updated model will keep a link for the agent named $\underline{a}$, if any disagreement between val and $v_{a l}^{\prime}$ does not concern a neighbor of $\underline{a}$. Or, equivalently, if all neighbors of $\underline{a}$ are identical with respect to $G_{\ell}$. Precisely this condition is encoded in $Q\left(e^{v a l}, e^{v a l^{\prime}}\right)$.

To formally state that the dynamics of $\dagger \in \mathrm{D} \cup \mathrm{L}$ are simulated by $\Delta^{\dagger}$, the following clauses are added to translation $T_{\bullet}$, for $\bullet=x, y$ :

$$
\begin{aligned}
& T_{\bullet}([d] \varphi)=\left[\Delta^{d}, e^{d}\right] T_{\bullet}(\varphi), \\
& T_{\bullet}([\ell] \varphi)=\bigwedge_{e \in E^{\ell}}\left(\operatorname{pre}^{\ell}(e) \rightarrow\left[\Delta^{\ell}, e\right] T_{\bullet}(\varphi)\right)
\end{aligned}
$$

where $\left(\Delta^{\dagger}, e^{\dagger}\right)$ is an action model implementations of $\dagger \in \mathrm{D} \cup \mathrm{L}$. Then $\mathrm{KDL}$ statics and dynamics can be shown performable in DTML:

Proposition 2. For any finite agent hybrid network model $M$ with nominal valuation $g$ and $\varphi \in \mathcal{L}_{\mathrm{KDL}}: M, w, g(\bullet) \models \varphi$ iff $\mathrm{T}(M), w \models_{g} T_{\bullet}(\varphi)$, for $\bullet=x, y$.

Proof. By induction on $\varphi$. We include the cases for the dynamic modalities.

Let $\varphi=[d] \psi$, where $d=(\Phi$, post $)$. We need to show that

$$
M, w, g(x) \models[d] \psi \text { iff } \mathrm{T}(M), w \models_{g}\left[\Delta^{d}, e^{d}\right] T_{x}(\psi)
$$

(the case for $T_{y}$ is analogous). Note that $M, w, g(x) \models[d] \psi$ iff $M^{d}, w, g(x) \models \psi$ iff (by i.h.) $\mathrm{T}\left(M^{d}\right), w \models_{g} T_{x}(\psi)$. We will show that $\mathrm{T}\left(M^{d}\right)$ and $\mathrm{T}(M) \otimes \Delta^{d}$ satisfy the same formulas. To prove this, we will show that there is a bounded morphism linking these two models (it is straightforward to show that term-modal formulas are preserved when this is the case, as in the propositional modal setting). Define $b: \mathbf{T}\left(W^{d}\right) \rightarrow \mathbf{T}\left(W^{\Delta^{d}}\right)$ by $w \mapsto\left(w, e^{d}\right)$. We show that $b$ is a bounded morphism. 
1. $w$ and $\left(w, e^{d}\right)$ satisfy the same basic formulas:

$$
\begin{aligned}
& \mathrm{T}\left(M^{d}\right), w \models g \\
& \text { iff (i.h.) } \left.M^{d}, w, g(x) \models \mathrm{f} \doteqdot \mathrm{z}\right) \\
& \text { iff } M, w, g(x) \models[d] \mathrm{f} \doteqdot \mathrm{z} \\
& \text { iff (reduction axiom for }[d] \mathrm{f} \doteqdot \mathrm{z}) \\
& M, w, g(x) \models\left(\bigvee_{\varphi \in \Phi: p o s t(\varphi, \mathrm{f})=\mathrm{z}, \mathrm{z} \in \mathrm{Z}_{\mathrm{f}}} \varphi\right) \vee \\
& \qquad\left(\neg\left(\bigvee_{\varphi \in \Phi: \operatorname{post}(\varphi, \mathrm{f})=\mathrm{z}, \mathrm{z} \in \mathrm{Z}_{\mathrm{f}}} \varphi\right) \wedge \mathrm{f} \doteqdot \mathrm{z}\right)
\end{aligned}
$$

iff (i.h., where we let $g(x)=a$ for some $a \in A$ named $\underline{a}$ )

$$
\begin{aligned}
\mathrm{T}(M), w \models_{g} T_{x}( & \left(\bigvee_{\varphi \in \Phi: \operatorname{post}(\varphi, \mathrm{f})=\mathrm{z}, \mathrm{z} \in \mathrm{Z}_{\mathrm{f}}} \varphi\right) \vee \\
( & \left.\left(\left(\bigvee_{\varphi \in \Phi: \operatorname{post}(\varphi, \mathrm{f})=\mathrm{z}, \mathrm{z} \in \mathrm{Z}_{\mathrm{f}}} \varphi\right) \wedge \mathrm{f} \doteqdot \mathrm{z}\right)\right)(x \mapsto \underline{a})
\end{aligned}
$$

iff (by definition of $\left.\Delta^{d}\right) \mathrm{T}(M), w \models_{g} \operatorname{post}(e)\left(T_{x}(\mathrm{f} \doteqdot \mathrm{z})(x \mapsto \underline{a})\right)$

iff $\mathrm{T}(M) \otimes \Delta^{d},\left(w, e^{d}\right) \models_{g} T_{x}(\mathrm{f} \doteqdot \mathrm{z})(x \mapsto \underline{a})$

iff (since $g(x)=a$ and $a$ is named $\underline{a}) \mathrm{T}(M) \otimes \Delta^{d},\left(w, e^{d}\right) \models_{g} \mathrm{f} \doteqdot \mathrm{z}$.

2. if $(w, v) \in \mathbf{T}\left(\sim_{a}^{d}\right)$ then $\left(\left(w, e^{d}\right),\left(v, e^{d}\right)\right) \in \mathbf{T}\left(\sim_{a}^{\Delta^{d}}\right)$ :

$(w, v) \in \mathrm{T}\left(\sim_{a}^{d}\right)$ iff $(w, v) \in \sim_{a}^{d}$ iff $(w, v) \in \sim_{a}$ iff $(w, v) \in \mathrm{T}\left(\sim_{a}\right)$ iff $(w, v) \in$ $\mathrm{T}\left(\sim_{a}^{\Delta^{d}}\right)$ (since $\Delta^{d}$ does not change the accessibility relations).

3. if $\left(\left(w, e^{d}\right),\left(v^{\prime}, e^{d}\right)\right) \in \mathrm{T}\left(\sim_{a}^{\Delta^{d}}\right)$ then there is $v$ such that $(w, v) \in \mathbf{T}\left(\sim_{a}^{d}\right)$ and $b(v)=\left(v^{\prime}, e^{d}\right):$

Reasoning as in step 2, $\left(\left(w, e^{d}\right),\left(v^{\prime}, e^{d}\right)\right) \in \mathbf{T}\left(\sim_{a}^{\Delta^{d}}\right)$ iff $\left(w, v^{\prime}\right) \in \mathbf{T}\left(\sim_{a}^{d}\right)$, and $b\left(v^{\prime}\right)=\left(v^{\prime}, e^{d}\right)$.

Hence, $b$ is a bounded morphism, and $\mathrm{T}\left(M^{d}\right)$ and $\mathrm{T}(M) \otimes \Delta^{d}$ satisfy the same formulas. Thus, $M, w, g(x) \models[d] \psi$ iff $M^{d}, w, g(x) \models \psi$ iff (by i.h.) $\mathrm{T}\left(M^{d}\right), w \models_{g}$ $T_{x}(\psi)$ iff (bounded morphism) $\mathbf{T}(M) \otimes \Delta^{d},\left(w, e^{d}\right) \models_{g} T_{x}(\psi)$ iff $\mathrm{T}(M), w \models$ $T_{x}([d] \psi)$.

Next, let $\varphi=[\ell] \psi$. We need to show that

$$
M, w, g(x) \models[\ell] \psi \text { iff } \mathrm{T}(M), w \models_{g} \bigwedge_{e \in E^{\ell}}\left(\operatorname{pre}^{\ell}(e) \rightarrow\left[\Delta^{\ell}, e\right] T_{x}(\psi)\right)
$$

(the case for $T_{y}$ is analogous). Note that $M, w, g(x) \models[\ell] \psi$ iff $M^{\ell}, w, g(x) \models \psi$ iff (by i.h.) $\mathrm{T}\left(M^{\ell}\right), w \models_{g} T_{x}(\psi)$. As in the previous case, we will show that $\mathrm{T}\left(M^{\ell}\right)$ and $\mathrm{T}(M) \otimes \Delta^{\ell}$ satisfy the same formulas by defining a bounded morphism linking the two. Note that the preconditions in $\Delta^{\ell}$ are pairwise inconsistent and jointly exhaustive, since each precondition corresponds to one way of assigning truth values to the formulas in $G_{\ell}$. Hence, for each $w \in \mathrm{T}(W)$, there is exactly one event $e^{v a l}$ such that $\mathrm{T}(M), w \models \operatorname{pre}^{\ell}\left(e^{v a l}\right)$. Define $b: \mathrm{T}\left(W^{\ell}\right) \rightarrow \mathrm{T}\left(W^{\Delta^{\ell}}\right)$ by $w \mapsto\left(w, e^{v a l}\right)$. We show that $b$ is a bounded morphism.

1. $w$ and $\left(w, e^{v a l}\right)$ satisfy the same basic formulas:

This is clear from the fact that learning updates do not change the accessibility relations. $\mathrm{T}\left(M^{\ell}\right), w \models_{g} T_{x}(\mathrm{f} \doteqdot \mathrm{z})$ iff (i.h.) $M^{\ell}, w, g(x) \models \mathrm{f} \doteqdot \mathrm{z}$ iff $M, w, g(x) \models \mathrm{f} \doteqdot \mathrm{z}$ iff (i.h.) $\mathrm{T}(M), w \models_{g} T_{x}(\mathrm{f} \doteqdot \mathrm{z})$ iff $\mathrm{T}(M) \otimes \Delta^{\ell},\left(w, e^{v a l}\right) \models_{g}$ $T_{x}(\mathrm{f} \doteqdot \mathrm{z})$. 
2. if $(w, v) \in \mathbf{T}\left(\sim_{a}^{\ell}\right)$ then $\left(\left(w, e^{v a l}\right),\left(v, e^{v a l^{\prime}}\right)\right) \in \mathrm{T}\left(\sim_{a}^{\Delta^{d}}\right)$ :

As $\mathrm{T}(M)$ is weakly rigid, each agent has the same name in each equivalence class $[w]_{\sim_{a}}$ of $\sim_{a}$. In what follows, we let the name of any agent $o \in A$ in worlds of $[w]_{\sim_{a}}$ be $\underline{o}$. Now, $(w, v) \in \mathrm{T}\left(\sim_{a}^{\ell}\right)$ iff $w \sim_{a}^{\ell} v$

iff $w \sim_{a} v$ and $\forall b \in A\left(N_{w} a b \Rightarrow \forall \varphi \in \ell(M, w, b \models \varphi\right.$ iff $\left.M, v, b \models \varphi)\right)$

iff (contrapositive) $w \sim_{a} v$ and $\forall b \in A(\exists \varphi \in \ell((M, w, b \models \varphi$ and $M, v, b \models$ $\neg \varphi)$ or $(M, w, b \models \neg \varphi$ and $\left.M, v, b \models \varphi)) \Rightarrow \neg N_{w} a b\right)$

iff (by i.h.) $(w, v) \in \mathrm{T}\left(\sim_{a}\right)$ and (by def. of $\left.\mathrm{T}(M) \otimes \Delta^{\ell}\right)$

$\mathrm{T}(M), w \models_{g} \operatorname{pre}\left(e^{v a l}\right)$ and $\mathrm{T}(M), v \models_{g}$ pre $\left(e^{v a l^{\prime}}\right)$ for some val, val' $\in \mathcal{V}_{\ell}$, and for all $\underline{b} \in \mathrm{C}$ :

if there is a $\varphi \in \ell$ such that

$$
\begin{aligned}
& \left(\mathrm{T}(M), w \models_{g} T_{x}(\varphi)(x \mapsto \underline{b}) \text { and } \mathrm{T}(M), v \models_{g} T_{x}(\neg \varphi)(x \mapsto \underline{b})\right) \\
& \quad \text { or }\left(\mathrm{T}(M), w \models_{g} T_{x}(\neg \varphi)((x \mapsto \underline{b})) \text { and } M, v \models_{g} T_{x}(\varphi)(x \mapsto \underline{b})\right)
\end{aligned}
$$

then

$$
\mathrm{T}(M), w \models_{g} \neg N(\underline{a}, \underline{b})
$$

iff $(w, v) \in \mathrm{T}\left(\sim_{a}\right)$ and $\mathrm{T}(M), w \models_{g} \operatorname{pre}\left(e^{v a l}\right)$ and $\mathrm{T}(M), v \models_{g} \operatorname{pre}\left(e^{v a l^{\prime}}\right)$ for some val, val $\in \mathcal{V}_{\ell}$ and (by def. of $\left.\Delta^{\ell}\right) \mathrm{T}(M), w \models_{g\left[x^{\star} \mapsto a\right]} Q\left(e^{v a l}, e^{v a l^{\prime}}\right)$ iff $\left(\left(w, e^{v a l}\right),\left(v, e^{v a l^{\prime}}\right)\right) \in \mathbf{T}\left(\sim_{a}^{\Delta^{\ell}}\right)$.

3. if $\left(\left(w, e^{v a l}\right),\left(v^{\prime}, e^{v a l^{\prime}}\right)\right) \in \mathrm{T}\left(\sim_{a}^{\Delta^{\ell}}\right)$ then there is $v$ such that $(w, v) \in \mathrm{T}\left(\sim_{a}^{\ell}\right)$ and $b(v)=\left(v^{\prime}, e^{v a l^{\prime}}\right)$ :

Reasoning as in step 2, $\left(\left(w, e^{v a l}\right),\left(v^{\prime}, e^{v a l^{\prime}}\right)\right) \in \mathrm{T}\left(\sim_{a}^{\Delta^{d}}\right)$ iff $\left(w, v^{\prime}\right) \in \mathrm{T}\left(\sim_{a}^{\ell}\right)$, and $b\left(v^{\prime}\right)=\left(v^{\prime}, e^{v a l^{\prime}}\right)$.

Hence, $b$ is a bounded morphism, and $\mathrm{T}\left(M^{\ell}\right)$ and $\mathrm{T}(M) \otimes \Delta^{\ell}$ satisfy the same formulas. Thus, $M, w, g(x) \models[\ell] \psi$ iff $M^{d}, w, g(x) \models \psi$ iff (by i.h.) $\mathrm{T}\left(M^{\ell}\right), w \models_{g}$ $T_{x}(\psi)$ iff (bounded morphism) for the unique event $e^{v a l}$ such that $\mathrm{T}(M), w \models_{g}$ $\operatorname{pre}^{\ell}\left(e^{v a l}\right)$, we have $\mathrm{T}(M) \otimes \Delta^{\ell},\left(w, e^{v a l}\right) \models_{g} T_{x}(\psi)$ iff $\mathrm{T}(M), w \models_{g} \bigwedge_{e \in E^{\ell}}\left(\operatorname{pre}^{\ell}(e) \rightarrow\right.$ $\left[\Delta^{\ell}, e\right] T_{x}(\varphi)$ iff $\mathrm{T}(M) \models T_{x}([\ell] \psi)$.

This completes the proof.

With Prop. 2 embedding KDL in DTML, it remains to show that there is a complete and decidable system for the image of KDL. Up to translation, such a logic is then a logic for the class of KDL models. To state the result, denote the TML image of the class of $n$-agent KDL models by $\mathrm{T}\left(\mathrm{KDL}_{n}\right)$. We now define a set of formulas, $F_{n}$, which can be shown to characterise the class $\mathrm{T}\left(\mathrm{KDL}_{n}\right)$.

Definition 14. Let $\mathrm{F}_{n} \subseteq \mathcal{L}\left(\Sigma_{n}(\mathrm{FP}, N o m)+[\Delta]\right)$ be the logic extending the termmodal S5 logic with the reduction axioms for action models $\left(\Delta^{\dagger}, e^{\dagger}\right), \dagger \in \mathrm{D} \cup \mathrm{L}$ (defined in [1]), as well as the following static axioms: 
1. There are $n$ agents and they are all named:

$$
\begin{aligned}
\text { Named }_{\mathrm{n}}:=\exists x_{1}, \ldots, x_{n}( & \left(\bigwedge_{i, j \leq n, i \neq j} x_{i} \neq x_{j}\right) \wedge \forall y\left(\bigvee_{i \leq n} y=x_{i}\right) \wedge \\
& \left.\left(\bigwedge_{i, j \leq n, i \neq j} c_{i} \neq c_{j}\right) \wedge\left(\bigwedge_{i \leq n} x_{i}=c_{i}\right)\right)
\end{aligned}
$$

2. Weak rigidity (Def. 8):

$$
\operatorname{Rig}_{\mathrm{n}}:=\bigwedge_{c \in \mathrm{C}} \forall x\left((c=x) \rightarrow \forall y\left(K_{y}(c=x)\right)\right)
$$

3. The neighbour relation is irreflexive and symmetric:

$$
\text { Neigh }:=\forall x \forall y(\neg N(x, x) \wedge(N(x, y) \leftrightarrow N(y, x)))
$$

4. Agents know their neighbors: KnowNeigh $:=\forall x \forall y\left(N(x, y) \leftrightarrow K_{x} N(x, y)\right)$

We then obtain the result:

Proposition 3. $\mathrm{F}_{n}$ statically characterizes $\mathrm{T}\left(\mathrm{KDL}_{n}\right)$.

Proof. By model-checking of the formulas in $\mathrm{F}_{n}$.

Which we can use to state completeness:

Theorem 1. For any $n \in \mathbb{N}$, the logic $\mathrm{F}_{n}$ is sound, strongly complete and decidable w.r.t. $\mathrm{T}\left(\mathrm{KDL}_{n}\right)$.

Proof (sketch). By Prop. 3, $\mathrm{F}_{n}$ statically characterizes $\mathrm{T}\left(\mathrm{KDL}_{n}\right)$. The result then follows from three results from [1]: 1 . Any extension of the term-modal logic $\mathrm{K}$ with axioms $A$ is strongly complete with respect to the class of frames characterized by $A$, and 2 . If $A$ characterizes a class with finitely many agents, then the logic is also decidable, and 3. any dynamic DTML formula is provably equivalent to a static DTML formula using reduction axioms.

Thus, since $\mathrm{F}_{n}$ characterizes $\mathrm{T}\left(\mathrm{KDL}_{n}\right)$, which is a class with finitely many agents, and all dynamic axioms in $\mathrm{F}_{n}$ are probably equivalent to static DTML, it follows that $\mathrm{K}+\mathrm{F}_{n}$ is strongly complete and decidable with respect to $\mathrm{T}\left(\mathrm{KDL}_{n}\right)$.

\section{Final Remarks}

This paper has showcased DTML as a framework for modeling social networks, their epistemics and dynamics, including examples in which uncertainty about name reference and de dicto/de re distinctions are key to modelling information flow and network change correctly. It was shown that DTML may encode the popular hybrid logical models of epistemic networks, and that DTML may be used to obtain completeness for an open-question dynamics through emulation.

We are very interested in learning how DTML relates to GDDL with respect to the encodable dynamics. We have been able to emulate the updates used 
in the examples of [22], but the general question is open. Further, the statics of frameworks that describe networks using propositional logic $[2,14]$ must be DTML encodable, and we expect the name about their updates, where reduction axioms exist. This raises two questions: if we can show this by a general results instead of piecemeal, and whether principled DTML action models exist for classes of updates. E.g., the threshold update of [2] gives an agent's property $P$ if a given fraction of neighbors are $P$; for a fixed agent set, this is DTML encodable by using the reduction axioms of [2] to provide pre- and postconditions. For a principled update, however, seemingly we need a generalized quantifier (e.g., a Rescher quantifier). If so, the general update form is not DTML encodable. Classification results like these would add valuable insights on network logics.

\section{References}

1. A. Achen, A. O. Liberman, and R. K. Rendsvig. Dynamic Term-Modal Logics for Epistemic Planning. under review. arXiv:1906.06047, 2019.

2. A. Baltag and Z. Christoff. Dynamic Epistemic Logics of Diffusion and Prediction in Social Networks. Studia Logica, 2018.

3. A. Baltag and L. S. Moss. Logics for Epistemic Programs. Synthese, 139(2):165-224, 2004.

4. A. Baltag, L. S. Moss, and S. S. Solecki. The Logic of Public Announcements, Common Knowledge, and Private Suspicions. In TARK '98, p. 43-56. 1998.

5. J. van Benthem, J. van Eijck, and B. Kooi. Logics of communication and change. Information and Computation, 204(11):1620-1662, 2006.

6. T. Bolander. Seeing is Believing: Formalising False-Belief Tasks in Dynamic Epistemic Logic. ECSI 2014, volume 1283, p. 87-107.

7. T. Bolander and M. B. Andersen. Epistemic planning for single- and multi-agent systems. Journal of Applied Non-Classical Logics, 21(1):9-34, 2011.

8. T. Brauner. Hybrid logic and its proof-theory. Springer, 2011.

9. Z. Christoff. Dynamic Logics of Networks. PhD thesis, U. of Amsterdam, 2016.

10. Z. Christoff and J. U. Hansen. A two-tiered formalization of social influence. LORI 2013, volume 8196 of LNCS, 68-81. Springer, 2013.

11. Z. Christoff and J. U. Hansen. A Logic for Diffusion in Social Networks. Journal of Applied Logic, 13:48-77, 2015.

12. Z. Christoff, J. U. Hansen, and C. Proietti. Reflecting on social influence in networks. Journal of Logic, Language and Information, 25:299-333, 2016.

13. Z. Christoff and P. Naumov. Diffusion in social networks with recalcitrant agents. Journal of Logic and Computation, 29(1):53-70, 122018.

14. Z. Christoff and R. K. Rendsvig. Dynamic Logics for Threshold Models and their Epistemic Extension. Proc. of ELISIEM, 2014.

15. H. van Ditmarsch and B. Kooi. Semantic Results for Ontic and Epistemic Change. In Logic and the Foundations of Game and Decision Theory (LOFT 7), Texts in Logic and Games, Vol. 3, pages 87-117. Amsterdam University Press, 2008.

16. J. Hintikka. Knowledge and Belief: An Introduction to the Logic of the Two Notions. College Publications, 2nd, 2005 edition, 1962.

17. B. Kooi. Dynamic term-modal logic. In LORI 200\%, Texts in Computer Science 8, p. $173-185,2007$.

18. L. Zhen and J. Seligman. A Logical Model of the Dynamics of Peer Pressure. Electronic Notes in Theoretical Computer Science, 278(0):275-288, 2011. 
19. F. Liu, J. Seligman, and P. Girard. Logical Dynamics of Belief Change in the Community. Synthese, 191(11):2403-2431, 2014.

20. R. K. Rendsvig. Diffusion, Influence and Best-Response Dynamics in Networks: An Action Model Approach. In ESSLLI 2014 Student Session, p. 63-75. arXiv:1708.01477, 2014.

21. J. Seligman, F. Liu, and P. Girard. Logic in the Community. In Logic and Its Applications, p. 178-188. Springer, 2011.

22. J. Seligman, F. Liu, and P. Girard. Facebook and the epistemic logic of friendship. In TARK 2013, p. 229-238, 2013.

23. J. Seligman, F. Liu, and P. Girard. General Dynamic Dynamic Logic. In Advances in Modal Logic, vol. 9, p. 239-260. Springer, 2012.

24. S. Smets and F. R. Valezquez-Quesada. How to Make Friends: A Logical Approach to Social Group Creation. In LORI 2017, LNCS vol. 10455, p. 377-390, 2017.

25. S. Smets and F. R. Valezquez-Quesada. In Dynamic Logic. New Trends and Applications (DALI 2017), LNCS vol. 10669, p. 171-184. Springer, 2018. 